\title{
A importância de estratégias de enfrentamento ao estresse na terceira idade
}

\author{
The importance of stress coping strategies in the elderly \\ La importancia de las estrategias de afrontamiento del estrés en los ancianos
}

Recebido: 27/10/2021 | Revisado: 02/11/2021 | Aceito: 05/11/2021 | Publicado: 19/12/2021

\author{
Marcilene Nunes Abreu \\ ORCID: https://orcid.org/0000-0001-7010-927X \\ Faculdade Martha Falcão Wyden, Brasil \\ E-mail: marcileneabreu.psico@gmail.com \\ Samara de Oliveira Lima \\ ORCID: https://orcid.org/0000-0001-9218-1248 \\ Centro Universitário FAMETRO, Brasil \\ E-mail:samaradol_mara@ hotmail.com \\ Marilene Castilho de Almeida \\ ORCID: https://orcid.org/0000-0003-3959-1883 \\ Centro Universitário FAMETRO, Brasil \\ E-mail: marilene_castilho@hotmail.com \\ Sabrina de Oliveira Marques \\ ORCID: https://orcid.org/0000-0001-7062-6517 \\ Faculdade Martha Falcão Wyden, Brasil \\ E-mail: ipsi.sabrina@gmail.com \\ Sanara Macedo Sousa \\ ORCID: https://orcid.org/0000-0002-6799-8704 \\ Centro Universitário UNINORTE, Brasil \\ E-mail: sanaranara@hotmail.com \\ Rossivaldo Duarte de Lima \\ ORCID: https/orcid.org/00000-0002/9395-6997 \\ Faculdade Martha Falcão Wyden, Brasil \\ E-mail: rossivaldolima@ hotmail.com \\ Jorge Victor Araújo Gonçales \\ ORCID: https//orcid.org/0000-0003-0810-3808 \\ Universidade Luterana de Manaus CELM/ULBRA, Brasil \\ E-mail: jorge.gleice10@gmail.com \\ Israel Vitor Paulino de Carvalho \\ ORCID: https://orcid.org/0000-0002-3315-0377 \\ Universidade Luterana de Manaus CELM/ULBRA, Brasil \\ E-mail: israelvitor19@gmail.com \\ Gleice Pereira Lopes \\ ORCID: https:// rcid.org/0000-0002-4969-9491 \\ Faculdade Boas Novas, Brasil \\ E-mail: hector.mallu38@gmail.com
}

\begin{abstract}
Resumo
Este trabalho tem como objetivo fazer uma revisão bibliográfica sobre a relação entre o estresse e o idoso, enfatizar a importância de estratégias de enfrentamento ao estresse na terceira idade. A metodologia utilizada foram consultas de livros e artigos, busca on-line em plataformas de pesquisas como o SCIELO, PEPSIC, EBSCO e Google Acadêmico proporcionando ao leitor a compreensão sobre o que é o estresse, de a como o idoso são percebidos pela sociedade, sua relação no idoso, quais os possíveis fatores que podem desencadear-lo durante a velhice e por fim reforçar a importância de estratégias de enfrentamento adequadas no combate ao estresse na terceira idade. Pode-se perceber que o estresse é a resposta do organismo a uma situação de perigo que prepara o corpo para fugir ou lutar, essa ação causa tensão e irritação onde o organismo reage a componentes físicos ou psicológicos quando há uma situação que provoque medo, excitação ou confusão, podendo acometer qualquer pessoa, independentemente de sexo e idade, e muitos idosos queixam-se sobre situações extremamente estressoras que influenciam diretamente na sua forma de perceber e viver a vida, a maneira como esses eventos estressores são percebidos possibilitam muita das vezes uma percepção equivocada da situação, fazendo com que o idoso tenha uma resposta inadequada frente ao estresse. Foi através disso que se entende que, mudanças contínuas que ocorrem em diferentes graus e intensidades e que são inerentes ao desenvolvimento humano, sendo preditores de estresse na terceira idade, podendo assim prejudica-los de forma extrema.
\end{abstract}

Palavras-chave: Estresse em idosos; Preditores; Estratégias de enfrentamento. 


\begin{abstract}
This work aims to carry out a literature review on the relationship between stress and the elderly, to emphasize the importance of coping strategies for stress in the elderly. The methodology used were book and article consultations, online search in research platforms such as SCIELO, PEPSIC, EBSCO and Academic Google, providing the reader with an understanding of what stress is, and how the elderly are perceived by society, its relationship in the elderly, what are the possible factors that can trigger it during old age and, finally, reinforce the importance of adequate coping strategies in combating stress in old age. It can be seen that stress is the body's response to a dangerous situation that prepares the body to flee or fight, this action causes tension and irritation where the body reacts to physical or psychological components when there is a situation that causes fear, excitement or confusion, which can affect anyone, regardless of gender and age, and many elderly people complain about extremely stressful situations that directly influence their way of perceiving and living life misperception of the situation, causing the elderly to have an inadequate response to stress. It was through this that it is understood that continuous changes that occur in different degrees and intensities and that are inherent to human development, being predictors of stress in old age, which can harm them in an extreme way.
\end{abstract}

Keywords: Stress in the elderly; Predictors; Coping strategies.

\title{
Resumen
}

Este trabajo tiene como objetivo realizar una revisión de la literatura sobre la relación entre el estrés y las personas mayores, para enfatizar la importancia de las estrategias de afrontamiento del estrés en las personas mayores. La metodología utilizada fue la consulta de libros y artículos, la búsqueda online en plataformas de investigación como SCIELO, PEPSIC, EBSCO y Academic Google, proporcionando al lector una comprensión de qué es el estrés, y cómo la sociedad percibe a las personas mayores, su relación con las personas mayores., cuáles son los posibles factores que pueden desencadenarlo en la vejez y, finalmente, reforzar la importancia de estrategias de afrontamiento adecuadas en la lucha contra el estrés en la vejez. Se puede apreciar que el estrés es la respuesta del cuerpo ante una situación de peligro que lo prepara para huir o luchar, esta acción provoca tensión e irritación donde el cuerpo reacciona a componentes físicos o psicológicos cuando existe una situación que provoca miedo, excitación o confusión., que puede afectar a cualquier persona, independientemente del sexo y la edad, y muchas personas mayores se quejan de situaciones extremadamente estresantes que influyen directamente en su forma de percibir y vivir la vida percepción errónea de la situación, provocando que las personas mayores tengan una respuesta inadecuada al estrés. Es por ello que se entiende que los continuos cambios que se dan en diferentes grados e intensidades y que son inherentes al desarrollo humano, son predictores de estrés en la vejez, que puede perjudicarlos de forma extrema.

Palabras clave: Estrés en el anciano; Predictores; Estrategias de afrontamiento.

\section{Introdução}

O estresse tem sido um tema bastante estudado atualmente por diversas áreas, principalmente por profissionais da área de saúde, isto se dá ao fato da grande preocupação com as consequências que o estresse pode acarretar na qualidade de vida do indivíduo no decorrer de todas as fases da vida, principalmente durante na velhice. Muitas são as mudanças que ocorrem durante o ciclo vital, mudanças estas que podem ser fortes preditoras de estresse para a maioria dos idosos, por isso, saber identifica-los é importante para poder eleger as melhoras estratégias de enfrentamento ao estresse durante a terceira idade.

A pesquisa realizada neste trabalho é classificada como pesquisa de revisão bibliográfica, onde foram realizadas consultas em livros e artigos científicos através da busca on-line, utilizando plataformas de pesquisas como o SCIELO, Google Acadêmico, PEPSIC e EBSCO. A pesquisa bibliográfica é feita com o intuito de levantar um conhecimento disponível sobre teorias, a fim de analisar, produzir ou explicar um objeto sendo investigado. A pesquisa bibliográfica visa então analisar as principais teorias de um tema, podendo ser realizada com diferentes finalidades. (Chiara, et al., 2008).

Portanto, este artigo pretende proporcionar ao leitor primeiramente a compreensão sobre o que é o estresse e sobre como o idoso é percebido pela sociedade, em seguida será enfatizado a relação do estresse durante a velhice, além de descrever os preditores relevantes e por fim será abordado sobre a importância das estratégias de enfrentamento do estresse na terceira idade. 


\subsection{Conceito de Estresse:}

Desde a pré-história observou-se o reconhecimento de que o homem sofria inúmeros tipos de exaustão após o trabalho, como medo, exposição ao calor e frio, fome, doenças, dentre outras. Essas situações tendem a ocasionar alguns desfechos biológicos e psicológicos, conhecidos hoje como estresse. De modo que, devido à evolução do conceito de estresse ao longo dos anos, torna-se fundamental compreender o conceito da palavra estresse mediante a grande possibilidade da mesma poder ser aplicada em diferentes áreas de conhecimento e possibilitar conotações distintas de acordo com sua aplicabilidade (Silva et al., 2018).

O conceito de estresse foi publicado pela primeira vez por Hans Selye, que considerava o estresse como um elemento inerente a toda doença que produz certas modificações na estrutura e na composição química do corpo, sendo possível ser observado e mensurado. Sendo o estresse o estado que se manifesta através da Síndrome de Adaptação Geral (SAG), que compreende a dilatação do córtex da supra-renal, atrofia dos órgãos linfáticos e úlceras gastro-intestinais, assim como perda de peso e outras alterações. Ou seja, é um conjunto de respostas não específicas do corpo a qualquer demanda que ocorre em três fases: 1) Reação de alarme, onde o organismo é mobilizado para enfrentar a ameaça; 2) Resistência, quando as manifestações agudas desaparecem à medida que o corpo tenta se adaptar a emergência reabastecendo hormônios adrenais; 3) Exaustão, quando a situação estressante persiste e a resistência não é mais possível, geralmente nessa etapa as reservas de energia do corpo são exauridas, podendo haver o colapso do organismo (Santos et al., 2015).

De acordo com os estudos de Selye, os sintomas de cada fase surgem como resultados de respostas neuroendócrinas do organismo ao estressor, envolvendo os dois eixos principais: hipotálamo - hipófise - córtex da glândula supra-renal e hipotálamo - hipófise - sistema nervoso simpático - medula da glândula supra-renal. Onde a liberação dos diferentes hormônios que participam do estresse é medida por centros corticais e supracorticais, que estão relacionados às funções cognitivas, emocionais e comportamentais, fazendo com que a intensidade dos sinais de estresse varie de acordo com cada fase de reação.

Sendo que no primeiro eixo, o hipotálamo libera o hormônio de liberação de corticotrofina (CRH), estimulando a hipófise a aumentar a produção de hormônio adreno-corticotrófico (ACTH), e o ACTH passa a agir no córtex das glândulas supra-renais estimulando o aumento da síntese de corticoides (cortisol e aldosterona), onde o aumento desses hormônios ocasiona sintomas como por exemplo, alteração do peso corporal, osteoporose, distúrbios de comportamento, alterações do padrão de sono, dentre outros sintomas.

No segundo eixo, o sistema nervoso simpático, está sob a ação da hipófise que estimula a medula supra-renal a liberar catecolaminas na circulação sanguínea (adrenalina e noradrenalina), causando a dilatação da pupila, aumento da sudorese, frequência cardíaca e da força contrátil do miocárdio dentre outros efeitos. Vale salientar que os corticoides e catecolaminas são hormônios fundamentais na resposta fisiológica ao estresse e que os níveis de cortisol modulam o sistema imunológico, influenciando na resposta inflamatória aos estressores e nas reservas de energia do organismo. Esse modelo apresentado por Selye foi criticado por muitos pesquisadores da época, que ao contrário de Selye não estavam preocupados apenas com as respostas neuroendócrinas do estresse e sim com as respostas ao estresse que os indivíduos apresentavam mediante ao confronto com os eventos da vida (Silva et al., 2018).

Lipp (2000), também contribuiu significativamente para o entendimento a cerca do estresse, onde ampliou o modelo proposto por Selye, incluindo a fase de quase exaustão, situada entre as fases de resistência e exaustão, momento em que ocorre um enfraquecimento do organismo que não consegue se adaptar ou resistir ao estresse e acaba tornando-se vulnerável ao aparecimento de doenças. Ainda segundo os estudos de Lipp (1996), para melhor compreensão de como se manifesta o estresse, torna-se indispensável compreender o que é um estressor. É chamado de estressor tudo aquilo que causa a quebra 
da homeostase interna e que exige algum tipo de adaptação. Por isso, a resposta ao estresse é influenciada pela intensidade, duração e âmbito do estressor e pelo número de estressores presentes no momento.

Segundo Góis et al., (2004), o estresse é qualquer situação em que uma demanda não específica exige que o indivíduo reaja e tome uma atitude. Sendo uma situação tensa, fisiológica ou psicológica que pode afetar a pessoa em todas as dimensões humanas. Para Gazzaniga e et al., (2005), o estresse ocorre como consequências de situações que extrapolam a capacidade de reação e adaptação do organismo através de respostas comportamentais e físicas, tendo como objetivo manter a estabilidade do corpo. Corroborando com esses autores, Milsted et al., (2009), afirmam que o estresse também pode ser entendido como uma reação interna do organismo para enfrentar situações que são percebidas como ameaçadoras e que preparam o indivíduo para agir, enfrentar ou fugir dessas situações.

Para Stefano, Bonanato e Raifur (2013), o estresse advém de uma reação do organismo que confronta situações que estimulam emoções negativas, tais como irritação e medo, ou positivas, como felicidade. Ou seja, após um evento estressor o organismo é solicitado a reagir, onde essa reação gera estimulação emocional que pode ser classificada como positiva ou negativa para o organismo, essa classificação depende muito da forma como o evento estressor é percebido e interpretado pelo indivíduo.

Dessa forma, compreender que o estresse não deve ser considerado apenas um fenômeno negativo é essencial, pois muito se fala dos danos que o estresse pode causar, porém não devemos esquecer-nos que o estresse não é apenas algo ruim, e que possui uma função importante para o equilíbrio do organismo, dependendo da frequência que ocorre. Mais conhecido como o bom estresse (eustress), é aquele que nos impulsiona a uma resposta adaptativa pelo organismo a determinada situação ou estímulo e o mau estresse (distress), refere-se aos estímulos fortes que podem provocar prejuízos ao organismo. Ou seja, o eustress é quando há alguma tensão que ocorre dentro dos limites da normalidade, considerado como fonte de equilíbrio para o organismo; E o distress é quando ocorre alguma tensão que ocasiona o desequilíbrio desse mesmo organismo (Nodari et al., 2014).

Portanto, observa-se que a definição do conceito de estresse é bastante rica e que diversas áreas de atuações distintas estão interessadas nessa temática. Mediante a isso, pode-se compreender que o estresse é a resposta do organismo a uma situação de perigo que prepara o corpo para fugir ou lutar. E quando o indivíduo se depara com uma situação que avalie como perigosa, sente-se intimado a responder a essa situação, exigindo que o organismo responda de acordo com o estímulo eliciador, podendo ser entendido através de experiências que causam tensão e irritação onde o organismo reage a componentes físicos ou psicológicos quando há uma situação que provoque medo, excitação ou confusão, podendo acometer qualquer pessoa, independentemente de sexo e idade. Dessa forma, é de suma importância compreender o que é o estresse, suas causas e quais as melhores estratégias de enfrentamento de acordo com a idade (Torquato et al., 2010).

\section{$1.2 \mathrm{O}$ idoso na sociedade}

A estrutura da população brasileira passou por diversas mudanças, onde houve momentos da história em que ocorreu um crescimento natural acelerado, com número de jovens maior do que de idosos. Em outros momentos, houve queda no crescimento natural, onde a população oscilou muito quanto à estrutura etária. Atualmente, no Brasil a população idosa tem aumentado progressivamente, pois o envelhecimento populacional é um fenômeno natural irreversível e mundial. Segundo Inagaki (2008), para a Organização Mundial da Saúde (OMS), a idade considerada idosa é estabelecida conforme nível socioeconômico de cada nação.

Em países em desenvolvimento caracteriza-se como idoso aquele que tem 60 ou mais anos de idade. Nos países desenvolvidos, a idade se estende para 65 anos. O aumento da proporção de pessoas com 60 anos ou mais é um fenômeno mundial que se deve principalmente a uma redução nas taxas de fertilidade e aumento da expectativa de vida. As projeções 
estatísticas da Organização Mundial de Saúde (OMS), afirmam que até 2050, existirá no mundo aproximadamente dois bilhões de pessoas nessa faixa etária, sendo que $80 \%$ em países em desenvolvimento e como consequência disso, o Brasil será o sexto país do mundo com maior número de idosos (OMS, 2005).

Para compreender alguns dos processos que ocorrem durante a velhice, é importante entender o que é o envelhecimento e qual a imagem que este repercute na sociedade. Neri (2008) define o envelhecimento como um processo de mudanças universais que são reguladas pela genética de cada indivíduo. Sendo que a dimensão biológica do envelhecimento se refere à modificação no funcionamento biológico, desde as células até os órgãos. Onde, o idoso não vivencia apenas modificações no funcionamento biológico, mas também apresenta mudanças de ordem socioeconômicas e psicológicas que podem está relacionadas com dificuldades de encarar novos papéis, perda na motivação e planejamento do futuro, enfrentamento das perdas orgânicas, afetivas e sociais, dificuldade de se adaptar a novas situações, modificações de caráter psíquico, depressão, hipocondria, somatização, níveis baixos de auto-estima, dentre outros.

Para Porto e Rezende (2016), o envelhecimento é um processo dinâmico, não linear, progressivo, iniciado no momento da concepção e que representa um conjunto de mudanças morfológicas, bioquímicas, fisiológicas, funcionais e psicológicas que interferem na adaptação do indivíduo no ambiente que vive. Onde ocorre o declínio das funções de modo diferenciado em cada indivíduo, sendo que as condições de vida e trabalho ao qual esse indivíduo esteve submetido durante toda sua existência influenciam no ritmo em que esse declínio ocorre. Já as alterações fisiológicas podem levar esse indivíduo a um estado de dependência, representado por um processo dinâmico cuja evolução pode ser modificada, retardada ou mesmo prevenida diante de um ambiente e auxílio adequados.

Ainda de acordo com esses autores, o envelhecimento pode ser diferenciado em quatro idades que são: cronológica, que é contada a partir do nascimento; biológica, referente às alterações fisiológicas, hormonais, anatômicas e bioquímicas do organismo, que são determinadas pela herança genética e ambiental; social, que se referem às normas, crenças, estereótipos e eventos sociais; idade psicológica, que se refere às mudanças comportamentais causadas por transformações biológicas, sobre a idade psicológica é importante enfatizar que ela é individual e é influenciada por normas, expectativas sociais e aspectos de personalidade.

Historicamente, a velhice começou a ser tratada como uma etapa da vida marcada pela decadência física e ausência de papeis sociais a partir da metade do século XIX. Onde o avanço da idade era caracterizado como um processo contínuo de perda e de dependência, que acabava por associar diversas imagens negativas á velhice. Ou seja, o idoso não era bem visto na sociedade devido ser associado ao ser que adoece e tem perdas conforme envelhece. Porém, ainda nos dias atuais o envelhecimento continua tendo essa associação entre doenças e perdas, onde essas perdas são relacionadas à deterioração do corpo, declínio cognitivo e incapacidade e as doenças estão relacionadas ao afastamento, dependência e morte. A imagem negativa atribuída ao idoso ocorre pela grande ênfase na juventude, beleza, autonomia, independência e na habilidade de ser produtivo ou reprodutivo.

Portanto, ser velho assume uma conotação negativa, devido ser sempre remetido a perda de atributos que são extremamente valorizados pela sociedade e simultaneamente pelo próprio idoso. E por incrível que pareça, a maioria dos estereótipos negativos relacionados à velhice, são atribuídos pelos próprios idosos, que na maioria das vezes não se reconhecem como velho e falam da velhice como se não fizessem parte dela (Schneider et al., 2008).

Para Uchôa (2003), apesar das inúmeras associações negativas sobre a velhice, é possível encontrar em alguns estudos realizados em sociedade não ocidentais imagens positivas sobre a velhice e o envelhecimento, demonstrando que as ideias de deterioração e perda não são universais e sim culturais. As imagens sobre a velhice e o envelhecimento são resultados de uma construção social e temporal que são construídas com base em valores e princípios próprios da sociedade. Ou seja, a forma como o idoso será visto pela sociedade, depende da cultura que está perpetua. 
Ainda assim, as associações negativas relacionadas à velhice perpetuam com o passar dos anos, mesmo com a existência de recursos que possibilitem prevenir e retardar as doenças e como consequência desse pensamento a velhice é temida por muitas pessoas, que a veem como uma etapa detestável. Diante desses fatos, o processo de envelhecimento só pode ser compreendido a partir da relação que se estabelece entre os diferentes aspectos cronológicos, biológicos e sociais, onde essa interação se dar de acordo com as condições da cultura na qual o individuo está inserido (Schneider \& Irigaray, 2008).

Miranda et al. (2016), afirmam que o envelhecer não necessariamente significa adoecimento, a menos que exista doença associada durante esse percurso, de outra forma o envelhecimento está associado a um bom nível de saúde. Para esses autores o processo de envelhecimento remete problemas de saúde que desafiam os sistemas de saúde e de previdência social. E como consequência disso, alguns países tem buscado compreender o processo de envelhecimento populacional, procurando alternativas para manter seus cidadãos idosos socialmente e economicamente integrados e independentes. Mas para isso, é fundamental investir em ações de prevenção ao longo de todo o curso de vida, pois é inevitável a presença crescente de pessoas idosas na sociedade. Dessa forma, é necessário vencer o desafio de inserir o tema do envelhecimento populacional dentro das formulações das políticas públicas, para que possam ser implementadas ações de prevenção e cuidado direcionados as necessidades da população que está envelhecendo.

\subsection{Preditores de estresse na terceira idade}

Durante toda a nossa vida, vivenciamos inúmeras experiências que podem ocasionar estresse, na velhice não é diferente. Muitos idosos queixam-se nos consultórios sobre situações extremamente estressoras que influenciam diretamente na sua forma de perceber e viver a vida, a maneira como esses eventos estressores são percebidos possibilitam diversas percepções equivocadas da situação, fazendo com que o idoso tenha uma resposta inadequada frente ao estresse. Portanto, mudanças contínuas que ocorrem em diferentes graus e intensidades e que são inerentes ao desenvolvimento humano podem ser percebidas como fortes preditores de estresse na terceira idade (Zanelli, 2012).

De acordo com estudos que avaliam a frequência de eventos estressantes durante o envelhecimento, os preditores mais frequentes durante esse processo estão atrelados às mudanças que os idosos enfrentam relacionados aos problemas com a aposentadoria, perda de entes queridos como, por exemplo, à perda do cônjuge ou perda de um filho, dificuldades financeiras, novas demandas de papéis sociais e doenças crônicas como, por exemplo, o AVC (Acidente Vascular Cerebral), sendo estes os eventos que exerce maior influencia no desenvolvimento de estresse durante a velhice. Vale salientar que não são os únicos eventos preditores de estresse em idosos, porém são os mais citados nas literaturas consultadas (Fortes-burgos et al. 2009).

Para Zanelli (2012), as mudanças que ocorrem na transição para aposentadoria são inevitáveis, sendo que para alguns, essas mudanças representam um tempo de novas conquistas, de liberdade e de desenvolvimento pessoal, porém para outros é percebida como um período de enfraquecimento de significados atribuídos à vida, de sentimentos de inutilidade e autodesvalorização, de vazio e de solidão, tornando-se um período de turbulência. Devido o modo de vivenciar a aposentadoria ser atrelado aos interesses e motivações pessoais, ao grau de comprometimento com o papel profissional até então exercido, e à condição econômica do aposentado, de modo que, é necessário que ocorra um processo de reorganização por conta do afastamento das atividades usuais de trabalho. Por isso, os problemas decorrentes da suspenção das atividades estão vinculados a sentimentos de frustração e de impotência frente às transformações. Contudo, a tensão resultante de tais processos pode ser vivida de diferentes maneiras, que dependendo da forma que são experienciadas por estes idosos podem ser fortes preditores de estresse, ou seja, se o idoso lida de modo inapropriado com os estressores, a probabilidade de 
consequências nocivas pode ser bastante acentuada, porém se este consegue lidar de modo adequado é possível neutralizar prejuízos ou até favorecer o desenvolvimento individual, obtendo menor desgaste durante esse período de transição.

A perda de entes queridos como, por exemplo, a morte de um cônjuge ou de um filho também é vistos como preditos de estresse significativos para o idoso, podendo acarretar além do estresse, outros problemas de ordem emocional, tais como a ansiedade e depressão pelo fato de alguns idosos não conseguirem lidar com sentimentos de desamparo, tristeza, medo, ressentimento, dúvida e com outros estados emocionais negativos. Ou seja, as mortes de pessoas amadas podem ser interpretadas por alguns idosos como uma ameaça a continuidade da vida pelo fato de que essas mortes prenunciam a morte e a dependência do próprio idoso. Outro ponto importante refere-se às dificuldades financeiras que porventura podem ocorrer devido à aposentadoria ou doenças enfrentadas por parentes e até mesmo pelo próprio idoso, assim como as novas demandas sociais atreladas a perda de alguns papéis sociais relevantes para o idoso ou empobrecimento desses papéis por conta do afastamento das atividades usuais de trabalho (Fortes et al., 2009).

Santos et al. (2015) em um estudo sobre o Estresse percebido em pacientes idosos sobreviventes do AVC, descrevem que um evento como o AVC torna a vida dos seus sobreviventes bastante estressante devido à relação que se estabelece entre essas duas variáveis, tendo como resultado um conflito de identidade acompanhado de sentimento de ser incapaz de gerir sua própria vida, onde a perda total ou parcial da independência é uma das experiências de transição mais difíceis para estes sobreviventes idosos. Ou seja, a perda da autonomia e da independência para realizar as atividades da vida diária como consequência de doenças é um fator que está fortemente presente na vida dos idosos. Por esta razão, compreende-se o fato de que eventos que remetem a um possível quadro de enfermidade, dependência e falta de controle também são fortes representantes de altos níveis de estresse para os idosos durante o envelhecimento.

\section{Metodologia}

O método utilizado foi o de pesquisa do tipo exploratória- descritiva com a abordagem qualitativa e o método dialético, pois visando a complexidade a ser pesquisada e os vários aspectos que o cercam, através da investigação, reflexão e ao estudo das percepções e práticas dos sujeitos, tanto pesquisadores, como o fenômeno social, para ter um maior entendimento destes fenômenos a partir do contexto em que estes se encontram. (Bogdan et al., 1994)

A maneira como o indivíduo percebe determinada situação e a interpretação que este dá ao evento estressor são fatores importantes no desencadeamento da resposta ao estresse. As percepções podem variar de acordo com cada indivíduo, por exemplo, duas pessoas podem vivenciar o mesmo evento (aposentadoria ou a morte de um ente querido), porém tendem a ter níveis de respostas distintas, devido à forma de interpretação e percepção que tiveram sobre esse evento. É essa percepção e interpretação que são elaboradas pelo indivíduo que determinam à resposta de enfretamento frente a um evento estressor (Margis et al., 2003).

Saber manejar o estresse do cotidiano é necessário, principalmente durante a velhice. Existem estratégias, estilos de vida e métodos eficazes na prevenção, redução e controle do estresse, dentre eles podemos citar os tratamentos psicoterápicos e as atividades físicas. Dentre os tratamentos psicoterápicos a TCC (Terapia Cognitivo-Comportamental), tem sido uma terapia bastante recomendada para auxiliar no enfrentamento ao estresse na terceira idade, devido a sua metodologia e terapia diretiva, que leva em consideração as demandas atuais apresentadas pelo paciente, além de ter uma estrutura educativa e organizada que permite ao paciente participação ativa no processo de mudança cognitivo-comportamental. Além disso, a TCC utiliza-se também de técnicas cognitivas e treinos comportamentais, como por exemplo, ensaio comportamental e roleplay que visam uma melhora na qualidade de vida do paciente, ajustando às necessidades e anseios de quem busca mais do que auxílio, uma nova maneira de se ver e se posicionar no mundo (Beck, 1997). 
Dentre as técnicas da Terapia Cognitivo-comportamental muitos pacientes idosos relatam se beneficiar das técnicas de relaxamento e de respiração, que são técnicas que ajudam no equilíbrio e que promovem reestruturação cognitiva, proporcionando expressão emocional reguladora e o desenvolvimento de um repertório de comportamentos mais adaptativos frente a situações eliciadoras de ansiedade e de estresse, tendo como finalidade diminuir a função do sistema nervoso autônomo simpático e os neurohormônios do estresse. Outra técnica bastante utilizada com idosos é a psicoeducação que tem como objetivo informar ao idoso como o corpo funciona quando estamos estressados e o que fazer para lidar de maneira assertiva frente a eventos estressores, pois à medida que o idoso começa a conhecer melhor sobre o funcionamento do estresse e o que pode ser feito para evitar níveis altos de estresse, é possível que este tenha uma melhor adesão ao tratamento proposto na terapia (Willhelm et al.,2015).

A atividade física também tem sido grande aliada ao combate e redução do nível de estresse na terceira idade. A atividade física se apresenta como uma distração e uma forma de afastar pensamentos negativos e gerar bem-estar, além de se ter a convivência com outras pessoas, aumentando a interação social. Além disso, causa alteração na concentração de endorfinas e monoaminas, que podem agir sobre a depressão, diminuindo a ansiedade, a tensão e o estresse (Ferreira, et al., 2016).

De acordo com Freitas (2001), uma das atividades que pode ser realizada pelo idoso é o vôlei, que aproxima pessoas com objetivos em comum, promovendo uma consciência social duradoura e estabelecendo como fatores motivacionais a diminuição do estresse, melhora de condicionamento físico e aumento do contato social. Porém, sabe-se que para praticar voleibol é preciso ter flexibilidade, força, potência, agilidade e condicionamento aeróbio sendo assim, como praticar o voleibol com o idoso? Sepulchro et al. (2017), fizeram uma adaptação desse esporte para o idoso e conseguiram resultados satisfatórios com essa adaptação. $\mathrm{O}$ voleibol para o idoso foi adaptado para que este pudesse jogar numa força segura e prazerosa, tendo como objetivo proporcionar momentos recreativos e dinâmicos, utilizando-se de movimentos simples e adaptados do voleibol convencional que auxiliem na melhora da qualidade de vida de seus praticantes, possibilitando o desenvolvimento no aspecto cognitivo, fisiológico, motor e social dos jogadores idosos e consequentemente a diminuição do nível de estresse destes.

Baptista et al., (2003), afirmam que o uso de atividades físicas contribui tanto na redução do estresse como na melhora da imunidade orgânica, além de apontarem que o uso da psicoterapia combinada com as atividades físicas é muito mais eficaz do que cada uma realizada de forma separada.

\section{Resultados e Discussão}

As mudanças que os idosos enfrentam com a chegada da aposentadoria, perda de entes queridos (perda do cônjuge ou perda de um filho), dificuldades financeiras, novas demandas de papéis sociais e doenças crônicas como o AVC marcam de forma significativa o processo de envelhecimento, assinalando a passagem de um status evolutivo para outro, refletindo a passagem do tempo, sinalizando o desenvolvimento normal e anunciando o alcance ou o não-alcance de metas e expectativas individuais e sociais que tendem a confrontar o idoso, influenciando diretamente na forma como este idoso interpreta a velhice e de como enfrenta esses eventos que podem ser percebidos como fortes preditores de estresse na terceira idade (Fortes-Burgos et al., 2009).

Segundo os estudos a adaptação a estes resultados são de fundamental importância, pois trará uma melhor qualidade de vida ao idoso e promoverá um envelhecimento mais saudável. Vários estudos trazem a temática do idoso, dentre eles a questão da memória, mas importante destacar que são insuficientes diante da crescente população idosa no país, afirma Marques et al., (2021) 


\section{Conclusão}

A resposta ao estresse é nada mais que um resultado da interação entre características pessoais do indivíduo e as demandas do meio que este está inserido, ou seja, as discrepâncias entre o meio externo e interno e a percepção deste quanto a sua capacidade de resposta. Por toda a nossa vida passamos por diversas mudanças e situações que nos ocasionam estresse e durante o processo de envelhecimento não é diferente, o idoso passa a perceber que suas habilidades naturais e sensoriais começam a sofrer declínios com intensidade variável e com isso passa a não apresentar a mesma competência na realização de algumas tarefas, além disso, problemas atrelados à aposentadoria, perda de entes queridos (perda do cônjuge ou perda de um filho), dificuldades financeiras, novas demandas de papéis sociais e doenças crônicas (como, por exemplo, o AVC) também podem ser percebidos como fortes preditores para o desenvolvimento de estresse durante a velhice. Ou seja, é evidente que o estresse excessivo proporciona redução da saúde, da qualidade de vida e do bem-estar, podendo levar o indivíduo à morbidade e até à morte. Por isso, saber manejar o estresse do cotidiano é necessário, principalmente durante a velhice.

Portanto, utilizar métodos eficazes para combater o estresse na terceira idade é um trabalho que requer uma busca por estratégias adequadas que devem está de acordo com as necessidades de cada paciente, por isso é importante selecionar recursos que levem em consideração o contexto, a realidade e idade do paciente. No caso de pacientes idosos, é necessário que os psicólogos que trabalham com esse público estejam familiarizados sobre as mudanças cognitivas, os problemas de vida diária dos idosos, as características peculiares das psicopatologias e metodologias de avaliação válidas e confiáveis para que sejam alcançados resultados positivos no processo terapêutico.

\section{Agradecimentos}

Agradecemos à Deus que nos fornece suas sementes de cada dia derramando sua graça.

\section{Referências}

Baptista, M. R., \& Alves, A. S. (2003) A atividade física no controle do stress. 8(17).

Beck, J. S. (1997) Terapia cognitiva: teoria e prática. Artmed

Chiara, I. D. et al. (2008) Normas de documentação aplicadas à área de Saúde. Editora E-papers

Ferreira, M. M., Ferreira, E. F., Toledo, C. \& Oliveira, R. A. R. (2016) Relação da pratica de atividade física e qualidade de vida na terceira idade. Caderno Científico Fagoc de Graduação e Pós-Graduação, 1, 9-15

Fortes-Burgos, A. C. G., Neri, A. L. \& Cupertino, A. P. (2009) Eventos de vida estressantes entre idosos brasileiros residentes na comunidade. Estud. Psicol, 14(1), 69-75

Freitas, G. R. Um estudo sobre fatores que influenciam na prática do vôlei de praia entre adultos na Zona Sul do Recife PE Brasil. Dissertação para Mestrado Faculdade de ciências do Desporto e de Educação Física; Universidade do Porto.

Furaste, P. A, (2012) Normas e Técnicas para o Trabalho Cientifico: Explicitação das Normas da ABNT. Dáctilo Plus.

Gazzaniga, M. S., \& Heatherton, T. F. (2005) Ciência psicológica: mente, cérebro e comportamento. Artmed.

Gois, C. F. L. \& Dantas, R. A. S. (2004) Estressores em uma unidade pós-operatória de cirurgia torácica: avaliação de enfermagem. Rev. Latino-Am. Enfermagem, 12(1), 22-27

Inagaki, R. K. et. al. (2008) A vivência de uma idosa cuidadora de um idoso doente crônico. Revista Ciência, Cuidado e Saúde, 7

Lipp, M. E. N. Manual do Inventario de sintomas de stress para adultos de Lipp. Casa do Psicólogo

Lipp, M. (2000) 15 anos de Pesquisa sobre Stress no Brasil. Anais do $1^{\circ}$ Simpósio sobre Stress e suas Implicações.

Margis, R., Picon, P., Cosner, A. F., \& Silveira, R. O. (2003) Relação entre estressores, estresse e ansiedade. Revista Psiquiátrica do Rio Grande do Sul, $25(1), 65-74$

Milstted, J. G., Amorim, C., \& Santos, M. (2009) Nível de estresse em alunos de psicologia do período noturno. In Congresso Nacional de Educação da PUC do Paraná. 
Research, Society and Development, v. 10, n. 16, e594101622400, 2021

(CC BY 4.0) | ISSN 2525-3409 | DOI: http://dx.doi.org/10.33448/rsd-v10i16.22400

Miranda, G. M. D., Mendes, A. C. G., \& Silva, A. L. A. (2016) O envelhecimento populacional brasileiro: desafios e consequências sociais atuais e futuras. Revista Brasileira de Geriatria e Gerontologia, 19(3), 507-519

Neri, A. L. (2008) Palavras chave em gerontologia. Alínea.

Nodari, N.L., Flor, S. R. A., Ribeiro, A. S., Carvalho, G. J., \& Hayasida, N. M. A. (2014) Estresse, conceito, manifestações e avaliação em saúde: revisão de literatura. Revista Saúde e Desenvolvimento Humano. 2(1), 61-74

OMS - Organização Mundial da Saúde. Envelhecimento Ativo: uma Política de saúde. Organização Pan-Americana da Saúde

Porto, C. F., \& Rezende, E. J. C. Terceira idade, design universal e aging-in-place. Revista Estudos em Design, 24(1), 152-168

Marques, S. de O., Lima, S.de O., Sousa, S. M.., \& Almeida, M. (2021) Cognitive assessment in elderly infected with P. vivax malaria. Research, Society and Development, 10(3), e731031324710.33448/rsd-v10i3.13247..

Santos, A. F., \& Santos, M. A. (2015) Estresse e Burnout no Trabalho em Oncolofia Pediátrica: Revisão Integrativa da Literatura. Revista Psicologia: Ciência e profissão, 35(2) 437-456

Santos, E., Rodrigues, R., Marques, S., \& Pontes-Neto, O. (2015) Estresse percebido nos idosos sobreviventes do AVC após a alta hospitalar para casa Revista da Escola de Enfermagem da USP, 49(5), 797-803

Schneider, R. H., \& Irigaray, T. Q. (2008) O envelhecimento na atualidade: aspectos cronológicos, biológicos, psicológicos e sociais. Revista Estudos de Psicologia, 25(4), 585-593

Sepulchro, B. N., Gonçalvez, E. C., \& Filho, J. F. (2013) Fatores motivacionais que levam a pratica do voleibol de quadra adaptado á terceira idade. Revista Carioca de Educação Física, 12(1), 43-51

Silva, R. M., Goulart, C. T., \& Guido, L. A. Evolução histórica do conceito de estresse. Revista Sena Aires, 7(2), $148-156$.

Stefano, S. R., Bonanato, F. M., \& Raifur, L. Estresse em funcionários de uma instituição de Ensino Superior: diferenças entre gênero. Economia \& Gestão, 13(31), 73-92

Torquato, J. A., Goulart, A. G., Vicentin, P., \& Correa, U. (2010) Avaliação do estresse em estudantes universitários. Revista InterSciencePlace, 1(14), 140154

Uchôa, E. (2003) Contribuições da antropologia para uma abordagem das questões relativas à saúde do idoso. Cad. Saúde Pública,19(3), 849-853

Willhelm, A. R., Andretta, I., \& Ungaretti, M. S. (2015) Importância das técnicas de relaxamento na terapia cognitiva para ansiedade. Contextos Clínic, 8(1), 79-86

Zanelli, J. C. (2012) Processos Psicossociais, bem-estar e estresse na aposentadoria. Rev. Psicol., Organ. Trab., 12(3), 329-340 